\title{
APP GROW GREEN: un instrumento para la innovación docente en la formación ambiental
}

\section{Areas tematicas: Recursos tecnológicos de soporte al aprendizaje o Incorporación de Aps y ODS en la educación superior}

\author{
Carla M. Tudorie ${ }^{a}$, María Vallés-Planells ${ }^{b}$, Eric Gielen ${ }^{c}$, Francisco Galiana ${ }^{b}$ \\ ${ }^{a}$ Instituto de Tecnología de Materiales, ${ }^{b}$ Dpto.de Ingeniería Rural y Agroalimentaria, ${ }^{\mathrm{c}}$ Dpto. de Urbanismo, \\ Universitat Politècnica de València.
}

\begin{abstract}
Nowadays, it is impossible to teach and educate a new generation of students who lives connected to technology in the same way it was done before. A modern vision of learning requires the adaptation of classic teaching strategies and methods. This study proposes the introduction of a gamified mobile application as a technological resource to support learning, in order to promote environmental education and awareness among Vera campus students of Universitat Politècnica de València. GROW GREEN APP is an innovative tool, which has the potential to achieve educational and sustainability goals, beyond its mere entertainment. This gamification project aims to help students find out the local biodiversity through different routes of urban flora (trees and shrubs) and fauna (birds), experience the sustainable mobility, enjoy the benefits provided by green infrastructure elements and discover green strategies for urban resilience. Citizen awareness is another promising context for gamification, facilitating the participation of the university community in bird censuses. The implementation of GROW GREEN APP will promote the acquisition of specific and transversal competences, that will provide an added value to students and will make courses more attractive.
\end{abstract}

Keywords: gamification, learning, higher education, biodiversity, university community, bird monitoring, mobile application, citizen science.

\section{Resumen}

Hoy en día, resulta imposible enseñar y educar como antaño a una nueva generación de estudiantes que vive conectada a la tecnología. Una visión moderna del aprendizaje requiere la adaptación de estrategias y métodos clásicos de enseñanza. Este estudio propone la introducción de una app móvil lúdica como recurso tecnológico de apoyo al aprendizaje, que fomente la educación y la conciencia ambiental en los estudiantes del campus de Vera de la Universitat Politècnica de València. La APP GROW GREEN es una herramienta innovadora, que tiene el potencial de conseguir objetivos educativos y de sostenibilidad, más allá del mero entretenimiento. Este proyecto de gamificación desea ayudar a los estudiantes a conocer elementos de la biodiversidad local mediante diferentes rutas de flora (árboles y arbustos) y fauna (aves) urbana, experimentar la movilidad sostenible, disfrutar de los beneficios suministrados por elementos de infraestructura verde y descubrir estrategias verdes para la resiliencia urbana. El compromiso ambiental es otro contexto prometedor para la gamificación, facilitando la participación de la comunidad universitaria en censos de aves. La implantación de la APP GROW GREEN fomentará la adquisición de competencias específicas y transversales, que proporcionan un valor añadido a los estudiantes y otorga mayor atractivo al currículo docente. 
Palabras clave: gamificación, aprendizaje, educación superior, biodiversidad, comunidad universitaria, monitorización de aves, aplicación móvil, ciencia ciudadana.

\section{Introducción}

A lo largo del tiempo, el aprendijaze ha sido definido de diferentes formas. En primer lugar, el aprendizaje es considerado un "cambio relativamente permanente en la conducta como resultado de la experiencia". En segundo lugar, se supone que es un "cambio relativamente permanente en las asociaciones o representaciones mentales como resultado de la experiencia" (Ormrod, 2005). Existen similitudes entre las dos definiciones con respecto al cambio relativamente permanente atribuido a la experiencia de las personas relacionadas con la frecuencia de unos acontecimientos, pero existen también algunas diferencias. La diferencia se atribuye al sujeto del cambio como el resultado del aprendizaje. De esta forma se introducen dos tipos de teorías: conductismo y cognitismo. Las teorías conductistas se centran en el aprendizaje de conductas tangibles y observables, denominadas respuestas (Ormrod, 2005). Se trata de un cambio en la conducta, un cambio externo visible (p. ej. aciones que tienen la función de estimular el estudio, el esfuerzo y la pasión por el aprendizaje, como conseguir "premios" que pueden ser puntos acumulados para incrementar las notas o directamente una buena calificación y el reconocimiento por parte del profesor que ayuda al estudiante a incrementar el interés por su asignatura). Las teorías cognitivas se centran en los procesos de pensamiento implicados en el aprendizaje humano (p. ej. encontrar relaciónes entre elementos del ecosistema o entre las especies y su entorno natural y utilizar la memoria, memoria visual, para relacionar la nueva información con el conocimiento previo). El cognitivismo se enfoca en los cambios en las representaciones o asociaciones mentales del estudiante con el fin de utilizar estrategias instruccionales, como atender, codificar, transformar, ensayar, almacenar y localizar la información (Ertmer y Newby, 1993).

En la actualidad, el desarrollo socio-cultural y la educación imponen una visión moderna de los modelos de aprendizaje y la adquisición de conocimientos, adaptada a las necesidades de la nueva generación, acostumbrada principalmente a los juegos electrónicos, medios digitales y a las redes sociales. Además, se ha demostrado la dependencia de los estudiantes de los dispositivos electrónicos móviles, afirmando que estas tecnologías son fundamentales en sus vidas (Alcaide y De la Poza, 2019). En este sentido, la generación de los "millenials" también es conocida como la generación "G de Gamers", por un nuevo término anglosajón "gamification" (game=juego), que se ha introducido en los diccionarios españoles como gamificación o ludificación (Rodríguez y Santiago, 2015).

La gamificación ha sido adoptada por los docentes como una herramienta, que permite enseñar a sus estudiantes, experimentar y disfrutar de la realidad que les rodea, de una forma entretenida y práctica. Se puede considerar que la gamificación es un concepto clave en la nueva educación porque facilita, de una forma normal, el aprendizaje trasladando los elementos de diseño de juego en contextos ajenos no-lúdicos (Deterding et al. 2011). El aprendizaje mediante la gamificación representa un método proactivo. En la literatura sobre la utilización de la gamificación en los campos educativos se mencionan algunas referencias con excelentes resultados como Alcaide y De la Poza, 2019, Serna et al. 2016, y González, 2015. Se reconoce que, mediante los juegos fuera de las aulas, los conocimientos se enriquecen y la participación y la motivación aumentan (Serna et al. 2016), incluso hasta inculcar a los estudiantes el sentimiento de sentirse útiles para la ciencia, gracias a sus esfuerzos personales.

En el contexto ambiental, se ha demostrado el potencial de aprendizaje de este tipo de herramientas. Por ejemplo, la app BIOTRACKER, que se ha desarrollado en el ámbito de la ciencia ciudadana en la fenología de las plantas (Bowser et al. 2013), o los ecopuzzles y evopuzzles desarrollados como una 
nueva metodología activa para la enseñanza-aprendizaje de las relaciones ecológicas y evolutivas (González, 2015).

La educación ambiental y el desarrollo de la conciencia ambiental son fundamentales para comprender la complejidad de la naturaleza, las relaciones sinérgicas del ecosistema urbano y los problemas contemporáneos de resiliencia y sostenibilidad de las ciudades.

La conciencia ambiental es un concepto multidimensional que incluye componentes cognitivos, actitudinales y conductuales (Schlegelmilch, Bohlen y Diamantopoulos, 1996). Los comportamientos individuales que contribuyen a la sostenibilidad ambiental forman parte de los comportamientos proambientales (Ones et al. 2015). Otros autores (Jiménez-Sánchez y Lafuente, 2010) añaden el aspecto afectivo, relacionado con las emociones y con los sentimientos de preocupación por el medio ambiente como la cuarta dimensión de la conciencia ambiental.

La educación ambiental es considerada una herramienta de sensibilización ambiental (Mesen, 2019) y se debe introducir en todos los ámbitos de la educación, consolidándose -llegando a su cenit- en las instituciones de educación superior. Las universidades, los institutos superiores o academias de formación técnica representan el epicentro de la innovación y del pensamiento crítico. Su función es crear nuevas generaciones de líderes, políticos, empresarios, científicos, investigadores y educadores. Las instituciones de educación superior son responsables de crear conciencia y fomentar una mejor comprensión de los objetivos de desarrollo sostenible determinados en la Agenda 2030 (United Nations, 2018).

En este trabajo, se propone la utilización de una herramienta eco-consciente como soporte del aprendizaje para los estudiantes del campus de Vera de la Universitat Politècnica de València (UPV). Su objetivo es mejorar el conocimiento y la sensibilización ambiental de los estudiantes a través de la gamificación y exploración de los elementos que constituyen la infraestructura verde urbana.

\section{Objetivos}

En el presente contexto, el objetivo de este trabajo es presentar la APP GROW GREEN y su potencial aplicación como recurso tecnológico de apoyo al aprendizaje para la comunidad universitaria. Esta APP permite:

1. Fomentar el aprendizaje y sensibilidad de los estudiantes sobre la biodiversidad local.

2. Involucrar a la comunidad universitaria en la identificación de las especies vegetales y en el censo de aves y promover la ciencia ciudadana.

3. Dar a conocer la importancia de las "soluciones basadas en la naturaleza" dentro de las ciudades y promover la participación de los alumnos y profesores en su seguimiento.

\section{Desarrollo de la innovación}

\subsection{Definición de los requisitos funcionales de la aplicación}

Grow Green es un proyecto europeo, financiado por H2020 (Grow Green, 2020), que tiene como objetivo principal aportar evidencias sobre los beneficios generados por "las soluciones basadas en la naturaleza" (NBS) a través del reconocimiento de los efectos ambientales de la ejecución de proyectos piloto en ciudades, entre las que se encuentra Valencia. La APP GROW GREEN surge, por tanto, como una herramienta que permita que la población se involucre en el seguimiento de las acciones piloto $\mathrm{y}$, en 
concreto, las desarrolladas en el distrito de Benicalap. Pero, como parte del proyecto europeo Grow Green, se pretende mejorar el conocimiento existente sobre los beneficios generados por los nuevos elementos de infraestructura verde urbana introducidos como proyectos piloto en el distrito. En este sentido, la app tiene una doble funcionalidad: didáctica y científica.

En relación a la función didáctica, se plantea como una herramienta que sirva para ampliar los conocimientos de los ciudadanos sobre la infraestructura verde urbana y su percepción sobre sus beneficios, contemplando como aspectos principales la biodiversidad local (vegetación arbórea y aves) y en las soluciones basadas en la naturaleza implementadas.

La funcionalidad científica implica proporcionar a la ciudadanía una herramienta que les permita colaborar voluntariamente con los gestores de la infraestructura verde y la comunidad científica en el seguimiento del censo de aves. Esta parte va dirigida a aquellos ciudadanos que muestren una mayor disposición a la participación, conocimiento y sensibilidad hacia su entorno y el bienestar de la comunidad.

\subsection{El diseño de la APP GROW GREEN}

\subsubsection{Antecedentes de la APP GROW GREEN}

La APP GROW GREEN está planteada desde el punto de vista de la dinámica de una ecogymkhana, basándose en el formato de otras apps gamificadas desarrolladas por la empresa PLAY \& GO EXPERIENCE, responsable del desarrollo informático de APP GROW GREEN. Algunos ejemplos de aplicaciones son: FALLAS 2018 TODO EL AÑO, LA TOMATINA 2018, WINE \& GO, VINOS Y BODEGAS DE VALENCIA, PEÑÍSCOLA LIVE THE GAME y FERROL DE LA ILUSTRACIÓN. Se trata de apps que, de una forma lúdica, incluyen una guía informativa de ciudades o de diferentes eventos, como el cineturismo y etnoturismo (Play \& go experience, 2020).

Conforme la definicion ofrecida por la Real Academia Española, la yincana es una" adaptación gráfica de la voz anglo-hindú gymkhana, conjunto de pruebas de destreza o ingenio que se realiza por equipos a lo largo de un recorrido, normalmente al aire libre y con finalidad lúdica". A través de la app GROW GREEN, los usuarios pueden seguir diferentes rutas (con temática ecológica) y realizar "las misiones propuestas". La app utiliza técnicas de geolocalización, realidad aumentada y gamificación, logrando así mejorar la experiencia del usuario.

Por otra parte, los contenidos de la APP GROW GREEN se han inspirado en apps de biodiversidad existentes como ARBOLAPP, BOTANY BUDDY Y PLANTNET, que sirven para la identificación de plantas, y EBIRD MOBILE, BIRDSEYE BIRD FINDING GUIDE Y BIRDS CENSUS, que son conocidas por la información proporcionada sobre las aves.

\subsubsection{Estructura de la APP GROW GREEN}

La APP GROW GREEN contiene tres módulos principales (figura 1). El mapa 2D indica la localización en tiempo real del usuario, el modo juego introduce al usuario como avatar en el mundo 3D y un módulo que fomenta la concienciación ambiental mediante avistamiento de aves. A través de diferentes rutas, se propone al usuario recorrer una serie de puntos de interés (POIs) que aparecen en el mapa 2D. Los POIs son las especies de árboles, arbustos, aves urbanas y periurbanas, selecionados previamente para representar las especies de flora y fauna (aves) más comunes de Benicalap y Valencia, así como las NBS implementadas en el contexto del proyecto europeo Grow Green. Cada POI se compone de distintos minijuegos para que el usuario interaccione con el entorno. Cumpliendo todos los minijuegos vinculados al POI, se completa la misión de la ecogymkhana. 


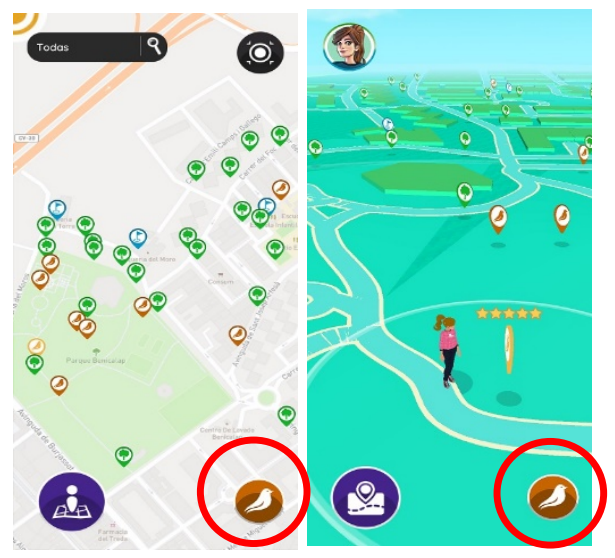

Fig. 1. Los módulos principales de la APP GROW GREEN: mapa 2D (izquierda), mundo 3D, (derecha) y censo de aves. El tercer módulo, censo de aves, está representado por un pictograma con la silueta de un ave en la parte derecha de ambas fotos.

\subsubsection{Puntos de interés (POIs) y rutas}

Las rutas pueden ser elegidas usando filtros de búsqueda libre en función de los intereses, las preferencias y necesidades del usuario. Las opciones de búsquedas ofrecidas por la app son: ver todos los elementos, o elegir los puntos clasificados por criterios (figura 2).

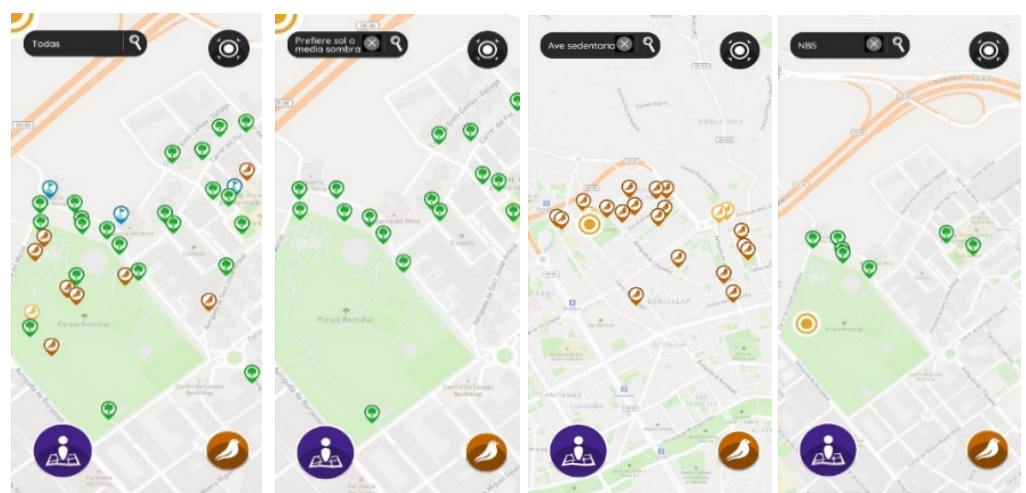

Fig. 2. Búsquedas de especies por rutas considerando (de izquierda a derecha: ningun criterio, las especies de vegetacion en función de sus necesides de luz, las aves sedentarías y las soluciones basadas en la naturaleza (NBS)

Los filtros de busqueda son diferentes según el tipo de POI y la información contenida. En el caso de la vegetación, se precisan los siguientes datos: nombre común (en castellano, valenciano e inglés), nombre científico, familia, crecimiento, curiosidades, usos y las características según factores ecológicos mencionadas en la tabla 1 . 
Tabla 1. Filtros de búsqueda de los árboles y arbustos de la app

\begin{tabular}{ll}
\hline Factores ecológicos & Características \\
\hline \multirow{2}{*}{ Origen especie } & Especie autóctona \\
& Especie alóctona \\
& Hoja caduca \\
Tipo de hoja & Hoja semiperenne \\
& Hoja perenne \\
& Requiere humedad media \\
Humedad & Indiferente a la humedad \\
Iluminación & Prefiere sol o semisombra \\
& Pleno sol \\
& Prefiere sombra \\
\hline
\end{tabular}

En el caso de las aves, la información contenida en la app revela: el nombre común (en castellano, valenciano e inglés), el nombre científico, familia, morfología, curiosidades, hábitat, movimientos migratorios, alimentación, comportamiento y estado de conservación. Según los movimientos migratorios, las aves han sido clasificadas en dos grupos: migratorias y sedentarias.

Cada POI se visualiza con pictogramas diferentes, en función del elemento contenido (p. ej. un árbol, un ave) y tiene asociados los siguientes datos: posición en el mapa junto con la distancia en kilómetros $(\mathrm{km})$, el tiempo necesario (expresado en horas, minutos) para llegar al destino andando, recursos multimedia (fotos, vídeos, imágenes $360^{\circ}$ ), descripción de las especies y valoraciones de los usuarios. Las aves, al ser imposible colocar su ubicación en tiempo real, como en el caso de los árboles y arbustos, han sido asociadas a los diferentes elementos de infraestructura verde urbana (calles arboladas, parques, jardines, etc).

\subsubsection{Minijuegos}

La ecogymkhana está disponible en el mundo 3D, donde el usuario puede cumplir un número máximo de cuatro objetivos, asociados a cada POI. Este módulo le permite al usuario aprender e interaccionar con el entorno mediante distintos minijuegos (misiones). Cumpliendo todos los minijuegos vinculados al POI se completa la misión de la ecogymkhana. Al cumplir las misiones se obtienen puntos que permiten subir de nivel para estar en lo más alto de la clasificación. Además, se pueden conseguir trofeos (virtuales) para fomentar la descarga y uso.

Los tipos de minijuegos aparecen con el simbolo dorado en la parte central de la la figura 3 y son los siguientes:

a. Check-in, supone el desplazamiento real de los usuarios hasta los diferentes POIs.

b. Captura de items virtuales a lo largo de las rutas y luego lanzamiento hacia el objetivo.

c. Selfie, supone hacerse fotos con el entorno y compartirla con los amigos, como un nuevo canal de educación con el que llegar a todos los usuarios.

d. Quiz, que pone a prueba los conocimientos de los usuarios utilizando recursos multimedia como fotos o sonidos o texto como posibles respuestas. 


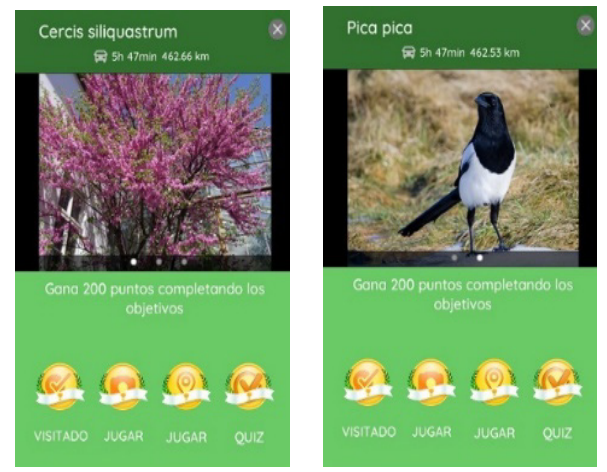

Fig. 3. Gamificación aplicada a dos ejemplos de POIs: árboles (árbol del amor o Cercis siliquastrum) y aves (Urraca o Pica pica)

\subsubsection{Avistamiento de aves}

Este módulo de la app trabaja mucho con la memoria visual. El fomento de la memoria visual es considerado útil en actividades prácticas de aprendizaje de aves urbanas (González, 2015). La aplicación incluye una lista de especies potenciales del distrito de Benicalap. Esta lista podría ampliarse a todas las especies presentes en la ciudad de Valencia. Se les propone a los usuarios mandar el número de individuos vistos tras haber pinchado las aves observadas mediante un listado de aves (pictogramas). Las aves se identifican por el vuelo, canto y sus características morfológicas peculiares p. ej. el petirrojo (Erithacus rubecula) por su mancha anaranjada en la del pecho (figura 4).
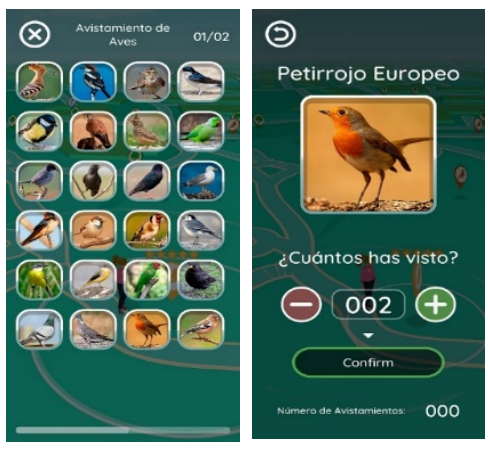

Fig. 4.Visualización del módulo relativo al censo de aves

\subsection{Selección de datos}

Para la ubicación de las especies de flora y fauna se ha empleado la capa de arbolado de Valencia (Ayuntamiento de Valéncia, 2018) y el mapa de infraestructura verde urbana (urban green infrastructureUGI) del distrito de Benicalap. Éste se desarrolló con la ayuda del sistema de información geográfica (Geographic Information System - GIS) y trabajo de campo. Para ello se utilizaron fotografias aéreas actualizadas de 2018 (resolución de $25 \mathrm{~cm}$ ) y el mapa catastral de Valencia. La aplicación, inicialmente, está diseñada para ser aplicada en el distrito de Benicalap. Sin embargo, tiene la posibilidad de extenderse a otras partes de la ciudad de Valencia.

Se han elegido 15 especies arbóreas nativas y un número mayor de especies foráneas o alóctonas (22), como consecuencia de la dominancia de especies introducidas en Valencia. De las especies nativas o autóctonas, 10 especies están presentes en las calles o en los parques de Benicalap y 5 representan futuras especies elegidas para la creación del bosquete climático, que representa uno de los proyectos pilotos de Grow Green. En la selección de nuevas especies introducidas se han considerado las necesidades ecológicas de las plantas relacionadas con un crecimiento óptimo, una gestión sencilla, especies con 
buena cobertura y que proporcionen un ambiente agradable para los ciudadanos mediante los valores estéticos. Para desarrollar el contenido de los POIs asociados a la vegetación, se ha utilizado a BallesterOlmos y Anguís (2001) como fuente de literatura de la flora urbana.

En cuanto las aves, se han selecionado las aves urbanas y periurbanas, que son fácilmente visibles en Valencia, completando con otros datos de la literatura existente de las aves de Valencia (García-Gans y Català, 2008) y trabajo de campo en el parque de Benicalap. La descripción de las aves se ha basado principalmente en la informacion proporcionada por la página web de la organización Seo BirdlifeSociedad Ornitológica Española (Seo Birdlife, 2019).

\section{Resultados esperados}

\subsection{Potenciales usuarios de la APP GROW GREEN en el contexto de la educación superior}

La APP está dirigida principalmente los estudiantes, ayudándoles adquirir y evaluar conocimientos y desarrollar competencias especificas y transversales en aquellas asignaturas que estén relacionadas con la botánica, la biodiversidad y el diseño de espacios verdes. En la tabla 2, se presenta una selección de las asignaturas ofertadas en la Universitat Politécnica de València en las que podría incorporarse esta herramienta.

Tabla 2. Asignaturas relacionadas con temas ambientales en la Universitat Politèctica de València

\begin{tabular}{lll}
\hline Escuela/Especialización & Asignaturas & Curso \\
\hline I. Escuela Técnica Superior de Ingeniería Agronómica y del Medio Natural & \\
Grado en Ingeniería Agroalimentaria y del Medio Rural & Botánica Sistemática & $2^{\circ}$ \\
& Flora Ornamental & $4^{\circ}$ \\
& Gestión de Fauna Silvestre & $4^{\circ}$ \\
& Jardinería y Paisajismo & $3^{\circ}$ \\
\hline Grado en Ingeniería Forestal y del Medio Natural & Bases de botánica forestal y zoología & $2^{\circ}$ \\
& Botánica sistemática & $2^{\circ}$ \\
& Diseño de espacios verdes & $4^{\circ}$ \\
\hline II. Escuela Técnica Superior de Arquitectura & & \\
Master Universitario en Arquitectura del Paisaje & Material vegetal & $1^{\circ}$ \\
\hline
\end{tabular}

Mediante el uso de la app en los espacios verdes urbanos se esperan los siguiente resultados:

- Suministrar un ambiente agradable para aprender

Para adquirir conocimientos ambientales ¿qué puede ser más agradable y más beneficioso que enseñar y aprender al aire libre? El interés y el uso de los espacios abiertos, como entorno de aprendizaje ha crecido mucho en los últimos años (Marcus y Wischemannr, 1990, Ibrahim y Fadzil, 2013, Hanan, 2013). Los beneficios para la salud, física y mental sumnistrados especialmente por la infraestructura verde han sido reflejados en la literatura científica (Ulrich et al. 1991, Lau y Yang, 2009, Gulwadi et al. 2019). La educación o la investigación en los espacios abiertos, el destrés, la salud mental y fisica han sido clasificados como servicios de paisaje culturales (Vallés-Planells, Galiana y Van Eetvelde, 2014, Tudorie et al. 2019). A través de la app, se transmiten conocimientos sobre la fauna y la flora de los espacios abiertos urbanos mediante la gamificación, un método lúdico de aprendizaje en un entorno sin preocupaciones, donde los estudiantes interaccionan y se relajan. Además, las rutas de la app están diseñadas para generar una movilidad sostenible, que los estudiantes puedan disfrutar en su recorrido, por 
ejemplo cuando cruzan espacios abiertos con buena cobertura verde o pasean por las calles arboladas, haciendo los trayectos visitados más agradables y curiosos por su diversidad.

- Contribuir al desarrollo de las funciones didácticas

Ferreiro (2012) recomienda a los profesores dedicar siete momentos (como unidad de tiempo) durante cada clase (espacio áulico) para cumplir las funciones didácticas, que son fundamentales para el aprendizaje. Estas funciones son: la recapitulación (momento R), la evaluación de lo aprendido (el momento E), la reflexión del proceso de aprendizaje y sus resultados, (momento de reflexión); el procesamiento de la información, (momento PI), la activación de los procesos neuro psicológicos que hacen posibles el aprendizaje (momento A), y la interdependencia con otros (momento I) y la orientación de la atención (momento O). La APP GROW GREEN puede contribuir al desarrollo de estas funciones.

En primer lugar, la app puede ayudar a captar el interés de los estudiantes por la materia y a mantenerlos activos durante las clases, clave para que se inicie el proceso de aprendizaje. Además, mediante las preguntas de los quiz para cada especie se realiza una recapitulación de los conocimentos adquiridos. Finalmente, la app se puede utilizar de forma que se fomenten la cooperación y la interdependencia positiva. Por ejemplo, el profesor puede diseñar diferentes actividades como algunos concursos de identificación de especies o de contestar muy rapido a las preguntas de quiz de forma individual (cada estudiante con su propio dispositivo) o por grupos. De esta manera, el estudiante comprende que su propio éxito está garantizado por el éxito de sus compañeros. A través de la gamificación, se puede conseguir fomentar las relaciones alumno-alumno, alumno-profesor y alumno-profesor-medio ambiente.

- Fomentar la adquisición de competencias específicas

La APP GROW GREEN se propone esencialmente como una herramienta de reconocimiento de especies y de exploración científica de las características morfológicas y funcionales. Mediante salidas de campo los estudiantes, por ejemplo de los primeros años de grado, aprenden a identificar correctamente las especies de flora y fauna urbana, propuestos como POIs en la app y solucionan los quiz temáticos. Los usuarios aprenden a asociar diferentes conceptos y elementos del ecosistema con las especies de flora y fauna. Observando las condiciones ecológicas del hábitat, las especies de vegetación se pueden estudiar según sus requisitos específicos, por ejemplo las especies que se desarrollan en pleno sol o especies que requieren humedad media, etc. En cuanto a la fauna, las aves urbanas están adaptadas a todos los tipos de medio, pero el comportamiento o el sonido de cada ave puede ofrecer varias pistas para su identificación. Además, las aves tienen alimentación diferente y pueden ser conectadas con algunas especies, concretamente por el tipo de fruta o de hábitat. Conforme Ausubel (1963), el aprendizaje requiere un esfuerzo cognitivo intencionado de relacionar la información a aprender con los esquemas o conocimientos previos que poseemos para lograr una nueva construcción cognitiva. Se considera que, mientras los usuarios pasan períodos de tiempo más largos dedicando interés a los árboles, arbustos y aves, ellos aprenden más eficazmente y más rápido los nombres comunes, científicos de las especies y también asociarlos entre sí o crear relaciones entre las denominaciones y las especies presentes en el distrito en tiempo real. Botánica sistematica, botánica forestal, zoología y gestión de fauna silvestre son ejemplos de asignaturas que usan los nombres científicos latinos o griegos, considerados por algunos estudiantes (Bowser et al. 2013) difíciles de memorizar.

- Fomentar la adquisición de competencias transversales

La UPV ha desarrollado un proyecto de competencias transversales para proporcionar a sus egresados un valor añadido que puede diferenciarlos de otros egresados y hacer más atractivos los estudios ofertados 
frente a ofertas similares de otras universidades (Universitat Politècnica de València, 2019). La APP GROW GREEN puede contribuir especialmente a dos de estas competencias:

- CT-07. Responsabilidad ética, medioambiental y profesional. El hecho de mejorar los conocimientos de los alumnos sobre la biodiversidad del entorno urbano que les rodea puede incrementar su sensibilidad y responsabilidad ambiental. Además, esta herramienta permite a la comunidad universitaria voluntaria ayudar a la administración local y la comunidad científica a monitorizar las aves. Se puede realizar un censo colaborativo de aves de los espacios abiertos.

- CT-10. Conocimiento de problemas contemporáneos. "Identificar e interpretar los problemas contemporáneos en su campo de especialización, así como en otros campos del conocimiento, prestando especial atención a los aspectos relacionados con la sostenibilidad tanto de la especialización propia como en otros contextos más amplios". Los estudiantes aprenden sobre medidas innovadoras para mejorar el entorno físico. La adquisición de esta competencia es de especial interés para los estudiantes de últimos años de grado y de máster. Como estrategias de mejora se introducen las NBS, que están pensadas para ser implementadas en este distrito en el 2020. Conocer la importancia de unas nuevas herramientas sostenibles, junto con la estructura, las funciones de las soluciones sostenibles es fundamental para los entornos urbanos comprometidos con el medioambiente y con el desarrollo sostenible. En el caso del distrito de Benicalap, las NBS son: el Corredor. Azul-Verde, Pavimento sostenible (Sistemas Urbanos de Drenaje Sostenible (SUDs)), Jardín vertical (ya implementado) y el Bosquete Climático. En la app estas soluciones verdes aparecen como POIs.

\section{Conclusiones}

Considerando el importante papel que juegan las instituciones de educación superior en la creación de la conciencia ambiental y fomento de aprendizaje de los estudiantes, se han analizado los resultados esperados tras haber propuesto la app sobre la biodiversidad APP GROW GREEN como recurso tecnológico de soporte al aprendizaje. Se ha llegado a la conclusión que, mediante la gamificación, los profesores pueden lograr combinar con éxito estrategias y métodos que consideran ambas teorías de aprendizaje, el cognitivismo y el conductismo, para optimizar la enseñanza de sus alumnos.

El uso de la app puede ser muy interesante para las asignaturas relacionadas con el estudio de la biodiversidad, los espacios abiertos y los componentes de la infraestructura verde, que son ofrecidas por los programas de estudios seleccionados. Se sugiere que la APP GROW GREEN puede sensibilizar a los estudiantes sobre la biodiversidad local, mientras que sus conocimientos se adquieren rápidamente y se quedan en la memoria a largo plazo. Los recursos multimedia de la app estimulan la memoria visual, considerada un factor clave en la fijación de información.

El concepto innovador de soluciones basadas en la naturaleza y su aplicación práctica en el distrito de Benicalap (Valencia) promueven la participación de la comunidad universitaria en su seguimiento. La gamificación llega a este nuevo público preparándoles para contribuir en un futuro a la ciencia ciudadana, a través de la monitorización de las aves urbanas. Además, se garantiza el disfrute de los beneficios ambientales, culturales y de suministro cuando los usuarios utilizan la app para recorrer rutas con criterio ecológico o explorar el mundo de la realidad aumentada. De esta forma los usuarios experimentan el potencial de la gamificación para su aprendizaje. 


\section{Referencias}

ALCAIDE, M.A. y DE LA POZA, E. (2019). "El uso de los dispositivos electrónicos móviles como herramienta docente de una asignatura de Grado" en Congreso de Innovación Educativa y Docencia en Red de la Universitat Politècnica de València (Congreso In-Red). Valencia: Universitat Politècnica de València. 110-120. $<$ http://dx.doi.org/10.4995/INRED2019.2019.10534> [Consulta:19 de febrero de 2020].

AUSUBEL, D.P.(1963). The Psychology of Meaningful Verbal learning. New York: Grune \& Stratton.

AYUNTAMIENTO DE VALENCIA (2018). Portal de transparencia y de datos abiertos. $<$ http://gobiernoabierto.valencia.es/es/dataset/?id=arbolado $>$ [Consulta: 18 de noviembre de 2019].

BALLESTER-OLMOS Y ANGUÍS, J.F. (2001). Arboles y arbustos de los jardines de Valencia. Valencia: Ayuntamiento de Valencia.

BOWSER, A., HANSEN, D., HE, Y., BOSTON, C., REID, M., GUNNELL, L. y PREECE, J. (2013). "Using gamification to inspire new citizen science volunteers" en Gamification '13: Proceedings of the First International Conference on Gameful Design, Research, and Applications. Stratford, Ontario, Canada. pp. 18-25. Disponible en $<$ https://doi.org/10.1145/2583008.2583011 > [Consulta: 19 de febrero de 2020].

DETERDING, S., SICART, M., NACKE, L., O'HARA, K. y DIXON, D. (2011). “Gamification: Using game design elements in non-gaming contexts" en CHI '11: CHI Conference on Human Factors in Computing Systems. Vancouver BC Canada. Disponible en<https://doi.org/10.1145/1979742.1979575> [Consulta:19 de febrerode 2020].

ERTMER, P.A. y NEWBY, T.J. (1993). “Conductismo, cognitivismo y constructivismo: una comparación de los aspectos críticos desde la perspectiva del diseño de instrucción” en Performance Improvement Quarterly, 6(4), pp. $50-72$.

FERREIRO, R. (2012). "La Pieza Clave del Rompecabezas del Desarrollo de la Creatividad: La Escuela” en Revista Iberoamericana sobre Calidad, Eficacia y Cambio en Educación, 10, 2, pp. 18-19. <

https://revistas.uam.es/index.php/reice/article/view/3068> [Consulta: 25 de febrero de 2020].

GARCÍA-GANS, F. J. y CATALÀ, F. J. (2008). Avifauna urbana y periurbana de la ciudad de Valencia, Monografías. Valencia: Ayuntamiento de Valencia. .

GONZÁLEZ, R. (2015). " Ecopuzzles/Evopuzzles: una nueva metodología activa para la enseñanza aprendizaje. (I) Interacciones ecológicas y (II) Relaciones evolutivas de los Tetrápodos" en Congreso de Innovación Educativa y Docencia en Red de la Universitat Politècnica de València (Congreso In-Red). Valencia: Universitat Politècnica de València. 11-25. Disponible en <http://hdl.handle.net/10251/84391> [Consulta: 20 de febrero de 2020].

GROW GREEN. GrowGreen, a partnership for greener cities to increase liveability, sustainability and business opportunities. < http://growgreenproject.eu/> [Consulta: 20 de febrero de 2020].

GULWADI, G.B., MISHCHENKO, E.D., HALLOWELL, G., ALVES, S. y KENNEDY, M. (2019). "The restorative potential of a university campus: Objective greenness and student perceptions in Turkey and the United States" en Landsc. Urban. Plan., 187, pp. 36-46, http://doi.org/10.1016/j.landurbplan.2019.03.003.

HANAN, H. (2013). "Space as Meaningful Place for Students in ITB Campus" en Procedia: Soc. Behav. Sci., 85, pp. 308 - 317. <http://doi.org/10.1016/j.sbspro.2013.08.361> [Consulta: 20 de noviembre de 2020].

IBRAHIM, N. y FADZIL, N.(2013).”Informal Setting for Learning on Campus: Usage and preference" en Procedia: Environ. Behav, 105, pp. 344 - 351. <http://doi.org/ 10.1016/j.sbspro.2013.11.036> [Consulta: 20 de noviembre de 2020].

JIMÉNEZ-SÁNCHEZ, M. y LAFUENTE, R. (2008). "Defining and measuring environmental consciousness" en Revista internacional de sociología (RIS), 68(3), pp. 2-4. <https://doi.org/10.3989/ris.2008.11.03>[Consulta: 24 de febrero de 2020].

LAU, S.S. y YANG, F. (2009). "Introducing Healing Gardens into a Compact University Campus: Design Natural Space to Create Healthy and Sustainable Campuses". Landsc. Res., 34(1), pp. 55-81. $<$ https://doi.org/10.1080/01426390801981720> [Consulta: 21 de febrero]. 
MARCUS, C.C. y WISCHEMANN, T.(1990). "Campus outdoor spacs" en Marcus, C.C. y Francis, C. People Places: Design Guidlines for Urban Open Space, 2nd ed. Cooper, M., C. Francis, C., Eds., Van Nostrand Reinhold: New York, USA 1990, pp. 143-170.

MESEN, D., (2019).” Teorías de aprendizaje y su relación con la educación ambiental Costarricense "en Ensayos Pedagógicos, 14, 1, p.187-202, < https://doi.org/10.15359/rep.14-1.8 > [Consulta: 20 de febrero de 2020].

ONES, D.S., WIERNIK, B.M., DILCHERT, S. y KLEIN, R. (2015). "Pro-Environmental Behavior” en International Encyclopedia of the Social \& Behavioral Sciences, 2nd ed., pp.82-88. <https://doi.org/10.1016/B978-0-08-0970868.22008-4> [Consulta: 24 de febrero de 2020].

ORMROD, J.E. (2005). "Definiciones y perspectivas del aprendizaje” en Ormrod, J.E. Introducción al aprendizaje humano (Human Learning (2005) trad.)( 4.a ed.). Posadas, J.L. Ed. Madrid: Pearson Education Hall.

PLAY \& GO EXPERIENCE. Catálogo de experiencias. < https://playgoxp.com/> [Consulta: 21de febrero de 2020]

REAL ACADEMIA ESPAÑOLA. Diccionario panhispánico de dudas.

$<$ http://lema.rae.es/dpd/?key=yincana>[Consulta: 21 de febrero de 2020].

RODRÍGUEZ, F. y SANTIAGO, R. (2015). "Qué es y qué no es la Gamificación. Un paseo por la Teoría de la Diversión” en Rodríguez, F. y Santiago, R. Gamificación: Cómo motivar a tu alumnado y mejorar el clima en el aula. Digital text. pp. 9-10. Disponible en <https:/www.researchgate.net/publication/299584812> [Consula:19 de febrero de 2020].

SEO BIRDLIFE-SOCIEDAD ESPAÑOLA DE ORNITOLOGÍA. Guía de aves. $<$ https://www.seo.org/> [Consulta: 20 de diciembre de 2019].

SERNA, E., MAURICIO, M.D., SAN MIGUEL, T. y MEGÍAS, J. (2016). "Experiencia de gamificación en Docencia Universitaria: aprendizaje activo y entretenido" en Congreso de Innovación Educativa y Docencia en Red de la Universitat Politècnica de València (Congreso In-Red). Valencia: Universitat Politècnica de València., pp. 364373. <http://dx.doi.org/10.4995/INRED2016.2016.4292> [Consulta:19 de febrero de 2020].

SCHLEGELMILCH, B.B., BOHLEN, G.M. y DIAMANTOPOULOS, A. (1996), "The link between green purchasing decisions and measures of environmental consciousness". European Journal of Marketing, Vol. 30,1996 (5) , pp. 35-55. < https://doi.org/10.1108/03090569610118740> [Consulta: 20 de febrero de 2020].

TUDORIE, C.M., GIELEN, E., VALLÉS-PLANELLS, M. y GALIANA, F. (2019). "Urban green indicators: a tool to estimate the sustainability of our cities" en Urban Agriculture 2018 1st International Conference on Urban Agriculture and City Sustainability. New Forest, UK. Wessex, UK. Disponible en < https://doi.org/10.2495/DNE-V0N0-1-11> [Consulta: 27 de febrero de 2020].

ULRICH, R.S., SIMONS, R.F., LOSITO, B.D., FIORITO, E., MILES, M.A. y ZELSON, M. (1991). "Stress recovery during exposure to natural and urban environments" en J. Environ. Psychol,11, pp. 201-230, $<$ https://doi.org/10.1016/S0272-4944(05)80184-7> [Consulta:19 de noviembre de 2019].

UNITED NATIONS. (2018). Mainstreaming Sustainable Development Goals into Higher Education Institutions in Small Island Developing States.

$<$ https://sustainabledevelopment.un.org/index.php?page=view\&type=20000\&nr=5157\&menu=2993 $>$ [Consulta: 20 de noviembre 2019]

UNIVERSITAT POLITÈCNICA DE VALÈNCIA. (2019). Competencias transversales. Valencia: Universitat Politècnica de València.

VALLÉS-PLANELLS, M., GALIANA, F. y VAN EETVELDE, V. (2014). "Classification of Landscape Services to Support Local Landscape Planning” en Ecology and Society, Vol, 19(1):44. <http://dx.doi.org/10.5751/ES-06251190144> [Consulta: 20 de enero de 2020] 